\section{Comparison of IOL power calculations by the IOLMaster vs theoretical calculations}

S Basu

Eye (2006) 20, 90-97. doi:10.1038/sj.eye.6701800;

published online 4 February 2005

Keywords: IOL power calculation; IOLMaster; measurement error; Hoffer Q.

\section{Introduction}

IOLMaster measures the axial length with the highest resolution and precision among the currently available diagnostic instruments for routine clinical use. ${ }^{1-3}$ At present, it gives options of using four theoretical formulas in its software, viz., Holladay I, Hoffer Q, SRK-T, and Haigis formulas. All of these well-known theoretical formulas apply the same principle for calculating the intraocular lens (IOL) power but vary in the method of calculating the predicted position of IOL postoperatively (predicted postop ACD), and the use of the number of predictor variables. This theoretical study was carried out to evaluate the agreement between the calculations as performed by IOLMaster and by a custom-made computer program according to the published methods of the respective formulas, namely, Holladay I, Hoffer Q, and SRK-T (the data using Haigis formula have not been included in this study because we are in the process of optimizing the required constants).

From a statistical viewpoint, a discrepancy or error in the yield of data from an instrument can be due to either a random error (due to a random variation around a fixed point), as determined by its precision, or a systematic error (due to a consistent over- or underestimation of the target point), as determined by the relative bias. ${ }^{4}$ Precision is determined by the techniques of measurement and was not the focus of this study. Instead this study focused on the relative bias of IOLMaster in its calculations.
Received: 28 July 2004 Accepted in revised form: 1 November 2004 Published online: 4 February 2005

\section{Department of} Kennington Road, Ashford, Correspondence: S Basu, Department of Kennington Road, Ashford, Kent TN24 OLZ UK 88534;

Fax: + 441233616770

E-mail: sbasu2003@

doctors.net.uk \begin{abstract}
Conclusion When using Hoffer-Q formula, the mean predicted postop SE with IOLMaster was $0.13 \mathrm{D}$ less than the computation in this study, that is, IOLMaster was showing a consistently myopic overestimation, or, a mean undercorrection of necessary intraocular lens power by $0.13 \mathrm{D}$, or, a residual hyperopic error of $0.13 \mathrm{D}$. This translates to a consistently more anterior estimation $(0.11 \mathrm{~mm}$ mean) of predicted postop ACD by IOLMaster, as compared with theoretical analysis.
\end{abstract}




\section{Method}

The present study was a theoretical analysis performed with hypothetical and identical data, which were input to IOLMaster $^{5}$ (here called the IM-set) and the published method of the respective formulas (here called the studyset). A computer program, written in Visual Basic programming language (code is given in Appendix A), was used for the theoretical calculations exactly in the way they were published in the original articles. ${ }^{6-8}$ The prescribed values (which vary according to the formula being used) of the corneal refractive index, retinal thickness factor, and secondary principal corneal plane (where appropriate) of the respective formulas were used. The value of ultrasonic axial length was converted to optical axial length as prescribed, where necessary. In the IOLMaster set-up screen, the Corneal R.I. was ensured to be 1.3375 , the standardised keratometric index of refraction.

For both data sets, the hypothetical and identical input variables were axial length (AL), average keratometry $(K)$, vertex distance, and lens constants for respective formulas. For each identical group of input variables, a set of IOL powers (range 14.0-26.5 D in 0.5 D steps) gave a corresponding set of predicted postoperative spherical equivalent (post-op SE) in both datasets, which were then compared, by calculating their differences (Figures 1 and 2). The range of IOL powers tested was used for the significance test, giving $n=26$.

In the next step, the predicted postop ACD was calculated, as the reverse solution of a quadratic equation, ${ }^{6}$ from the same set of input variables (AL, $K$, and vertex remaining the same, and each IOL power with its respective predicted postop SE yielding a

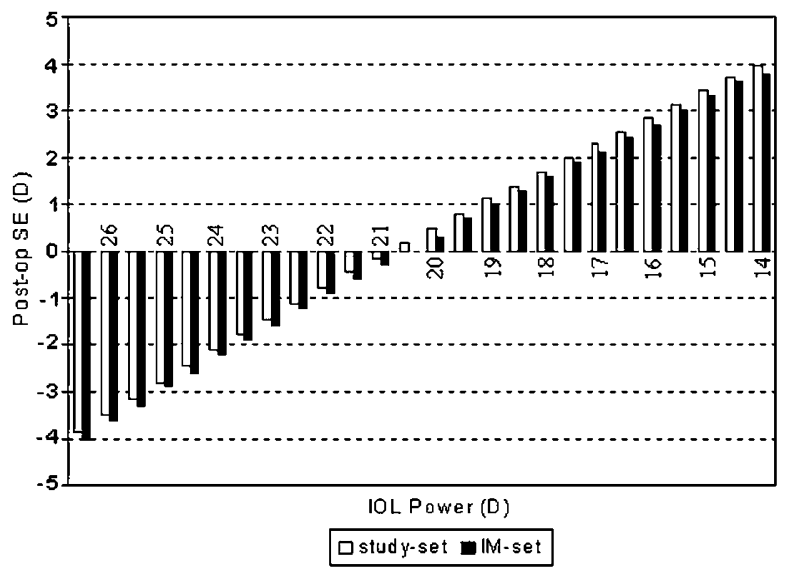

Figure 1 Comparison of predicted postop SE by varying IOL power from 14 to $26.5 \mathrm{D}$ (other predictor variables kept constant): IOLMaster set (IM-set $=$ dark bars) showed consistent myopic overestimation compared to the theoretical set (studyset $=$ light bars). corresponding predicted postop ACD value). This was performed first with the study-set data and then with the IM-set data and results are shown in Figure 3.

\section{Results with discussion}

All the theoretical formulas in context here use the refractive vergence formula for their calculations. There are six variables in the vergence formula: (1) net optical corneal power (derived from average keratometry), (2) optical axial length (derived from ultrasonic axial length), (3) IOL effective power, (4) predicted postop ACD, (5) predicted postop SE, and (6) vertex distance. The constants used are the refractive index of aqueous

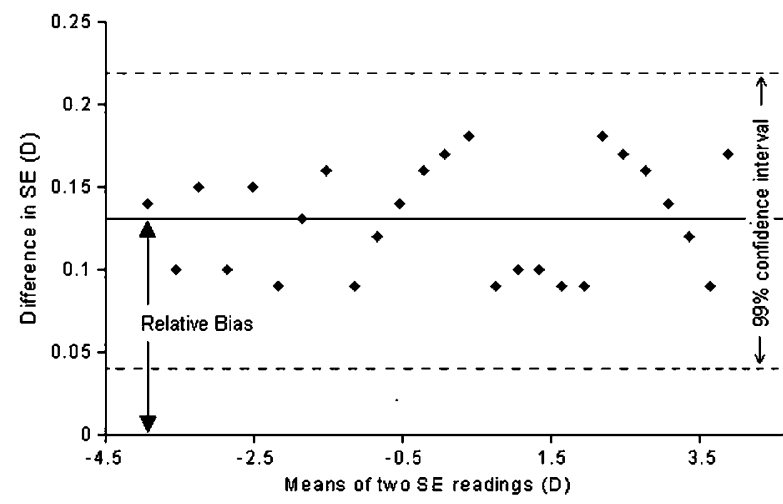

Figure 2 Difference $v s$ mean of pairs of SE readings: each of the pairs of predicted SE readings (IM-set and study-set) was taken and their difference plotted along the $y$-axis and the mean of the two along the $x$-axis. Characteristic of a systematic error, the variability of difference was unrelated to the magnitude of the SE values, all within the $99 \%$ confidence interval. Relative bias is the mean difference.

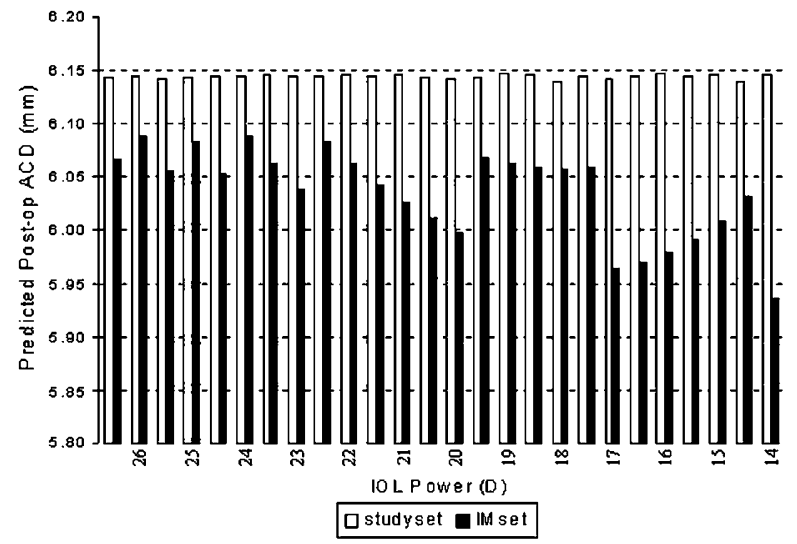

Figure 3 Comparison of predicted postop ACD for each pair of IOL power and postop SE as given in Figure 1 (again, other predictor variables kept constant): IOLMaster set (IM-set $=$ dark bars) showed consistently shorter prediction of ACD, as compared to theoretical set (study-set=light bars). Also note the wider data spread in the IM-set. 
and vitreous (1.336) and that of air (1.0). Since the same hypothetical input variables were used in both the studyset and IM-set, the accuracy of measurement of data and the optimisation of IOL constants were not relevant as confounding factors.

While keeping other variables constant, a set of IOL powers produced a corresponding set of predicted postop SE in each of the two data sets, which can now be compared between these data sets. Figure 1 shows the results of such data set pairs, using Hoffer-Q formula. A consistent discrepancy in the calculated postop SE between the two data sets was found when using Hoffer$Q$ formula but identical results were obtained when using Holladay I and SRK-T formulas. For an average eye with AL $23.45 \mathrm{~mm}$ and $K 45 \mathrm{D}$, and with ACD constant 6.15 and vertex of $12 \mathrm{~mm}$, the mean difference in the predicted postop SE between the study-set and the IM-set was $0.13 \mathrm{D} \pm \mathrm{SD} 0.03 \mathrm{D}$, and this difference was statistically significant at $1 \%$ significance level with the paired- $t$ test.

The mean difference is a measure for the systematic error (relative bias). For the same set of input variables, the difference in predicted postop SE between the two data sets is plotted against the mean of each pair (IM-set and study-set) of the postop SE (Figure 2), and the systematic error (relative bias) and the $99 \%$ confidence interval are shown. As the graph shows, the variability of systematic error was unrelated to the magnitude of the postop SE readings, as expected, and not significantly different (at 1\% level) from each other.

Since this difference was found only when using Hoffer-Q formula and not other formulas, the source of this discrepancy was thought to be lying in the prediction of the postop anterior chamber depth (predicted postop ACD), also called the Effective lens position, when using the Hoffer-Q formula. So in the next step, the predicted postop ACD was calculated, as reverse solution of a quadratic equation, as mentioned in the Methods section. Figure 3 shows the predicted postop ACD for corresponding IOL power and predicted postop SE for the two data sets. With the input data of an average eye (as mentioned above), the mean predicted postop ACD in the IOLMaster dataset (IM-set) was $0.11 \mathrm{~mm}( \pm S D$ $0.04 \mathrm{~mm}$ ) less than in the study dataset (difference statistically significant at $1 \%$ significance level with the paired- $t$ test). More importantly, the data spread of the predicted postop ACD was much higher in the IM-set (SD 0.042) than in the study-set (SD 0.002), indicating less consistency in estimation in the former set.

The accuracy of the calculations used in the study-set for the Hoffer-Q formula was verified by outputting in the computer program the value of the predicted postoperative $\mathrm{AC}$ depth, and comparing with the results published by Hoffer in his original article, ${ }^{7}$ and they were found to be in agreement. Also the fact that calculations when using other formulas gave identical results in the study-set and the IM-set verifies the program's accuracy used in this study.

To sum up, while using the Hoffer-Q formula, the mean difference in predicted postop spherical equivalent between the theoretical computation in this study and computation by IOLMaster was $0.13 \mathrm{D}( \pm \mathrm{SD} 0.03 \mathrm{D})$, with IOLMaster showing a consistently myopic overestimation, or in other words, a mean undercorrection of the necessary IOL power by $0.13 \mathrm{D}$, or equivalently, a residual hyperopic error of $0.13 \mathrm{D}$ by IOLMaster, as compared to the theoretical analysis. This translates to a consistently more anterior estimation ( $0.11 \mathrm{~mm}$ mean) of predicted post-op ACD by IOLMaster, as compared to theoretical analysis in this study. The resolution for the partial coherence laser interferometry is reported to be $12 \mu$ and precision $0.3-10 \mu^{1,2}$ while those of IOLMaster are documented to be 10 and $30 \mu$ (in human eye). ${ }^{3}$ It appears that this high degree of accuracy is rather compromised if the bias is not taken into account while performing IOL power calculations in IOLMaster.

Speculating about the source of this discrepancy between the study-set and that of IOLMaster, each of the five parts ${ }^{7}$ of the Hoffer-Q formula for prediction of postop ACD was considered. The two constant parts of the formula could not be the source because this would give a constant error of predicted postop ACD, unlike what was found here. The discrepancy was also found when $K$ was kept constant while AL varied and vice versa, or when AL value of 23.5 was used (which would have made the extreme axial length correction factor equal zero). Other constants like the retinal thickness factor and corneal index of refraction cannot be a source because they have been used as prescribed. By exclusion, it seems that the source of this discrepancy could possibly be a rounding-off error or a logical error in the calculations used by the IOLMaster.

It must be mentioned here that this study was theoretical in nature and the purpose was to test the performance of the computer program used by the IOLMaster; it has no bearing with actual clinical study, and hypothetical identical data sets were used for comparison. Hence, questions like the precision of biometry or keratometry, number of patients involved, or types of IOL inserted do not arise. Nor was there any intention to assess the clinical efficacy of the IOL formulas. Although numerous studies have been carried out to evaluate the precision in measurement by the IOLMaster, no previous study has focused on the purpose in context here. In this study, only data using the Hoffer-Q formula have been presented, because no discrepancy was found with Holladay I and SRK-T 
formulas (zero difference), as one would expect to obtain the same output from a formula when input data are the same. In this sense, applying the concept of statistical significance (and probability) is invalid if the problem is thought to be strictly in arithmetic functions; however, the origin of the problem is uncertain here. The program code is included in Appendix A for the interested reader.

\section{References}

1 Findl O, Drexler W, Menapace R, Hitzenberger CK, Fercher AF. High precision biometry of pseudophakic eyes using partial coherence interferometry. J Cataract Refract Surg 1998; 24: 1087-1093.

2 Baumgartner A, Moller B, Hitzenberger CK, Drexler W, Fercher AF. Measurements of the posterior structures of the human eye in vivo by partial coherence interferometry using diffractve optics. Proc SPIE 1997; 2981: 85-91.

3 IOLMaster Manual. Zeiss Meditech Inc., Software version 3.xx, 000000-1150-839.
4 Daly LE, Bourke GJ. Interpretation and Uses of Medical Statistics, 5th ed. Blackwell Science, Oxford, 2000.

5 IOLMaster version 3.01.0294. Carl Zeiss@ 1999, 2001.

6 Holladay JT, Musgrove KH, Prager TC, Lewis JW, Chandler TY, Ruiz RS. A three-part system for refining intraocular lens power calculations. J Cataract Refract Surg 1988; 14: 17-24.

7 Hoffer KJ. The Hoffer Q formula: a comparison of theoretic and regression formulas. J Cataract Refract Surg 1993; 19: 700-712. Nov. errata 1994; 20:677.

8 Retzlaff JA, Sanders DR, Kraff MC. Development of the SRK/ $\mathrm{T}$ intraocular lens implant power calculation formula. $J$ Cataract Refract Surg 1990; 16: 333-340 correction p 528.

\section{Appendix A}

The following data give a list of functions used in the standard module (technically called library) of the computer program, which may be called by various event procedures and subprocedures in other parts of the computer program. This list is the core of functions used in the program. Some variables may seem superfluous but are necessary to run other parts of the project.

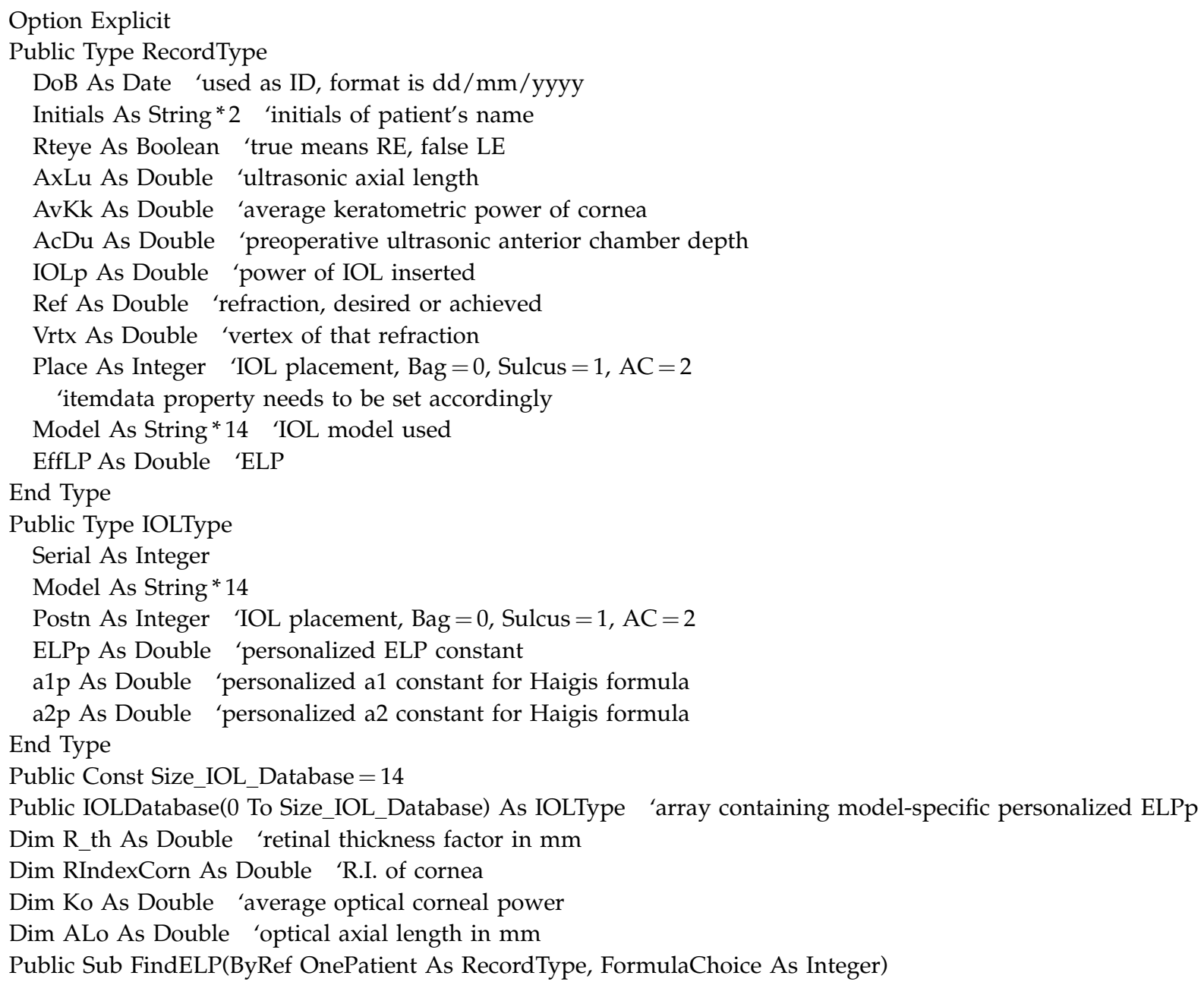


Dim X As Double, A As Double, B As Double, C As Double '4 constants in calculation With OnePatient

Select Case FormulaChoice

Case 1 'Holladay I

RIndexCorn $=4 / 3$

R_th $=0.25$

Const Pc2 $=0.05$

$\mathrm{ALo}=. \mathrm{AxLu}+\mathrm{R} \_$th-Pc2

Case 2 'HofferQ

RIndexCorn $=1.336$

$\mathrm{ALo}=. \mathrm{AxLu}$

Case 3 'SRK-T

RIndexCorn $=1.333$

R_th $=0.65696-0.02029 *$.AxLu

$\mathrm{ALo}=. \mathrm{AxLu}+\mathrm{R}$ th

Case 4 'Haigis

RIndexCorn $=1.3315$

$\mathrm{ALo}=. \mathrm{AxLu}$

End Select

$\mathrm{Ko}=. \mathrm{AvKk}^{*}((\mathrm{RIndexCorn}-1) /(1.3375-1))$

$X=1336 /((1000 /((1000 /$. Ref $)-$. Vrtx $))+$ Ko $)$

$\mathrm{A}=. \mathrm{IOLp}$

$\mathrm{B}=-. \mathrm{IOLp}{ }^{*}(\mathrm{ALo}+\mathrm{X})$

$\mathrm{C}=\left(1336^{*}(\mathrm{ALo}-\mathrm{X})\right)+\left(. \mathrm{IOLp}{ }^{*} \mathrm{X} * \mathrm{ALo}\right)$

If $(. I O L p>=0)$ Then

$. E f f L P=\left(-B-\operatorname{Sqr}\left(\left(B^{\wedge} 2\right)-(4 * A * C)\right)\right) /(2 * A)$

Else

'MsgBox "Invalid at present"

$. E f f L P=\left(-B+\operatorname{Sqr}\left(\left(B^{\wedge} 2\right)-(4 * A * C)\right)\right) /(2 * A)$

End If

MsgBox .EffLP

End With

End Sub

Public Sub FindPower (ByRef OnePatient As RecordType, OneIOL As IOLType, ELPpsnd As Double, FormulaChoice As Integer, PowerArray)

Dim ELPo As Double 'optimized ELP (depends on choice of formula)

Dim Offset As Double, X As Double, IOLtargetPower As Double

Dim Counter As Integer

With OnePatient

If $. \operatorname{Ref}=0$ Then

.Ref $=-0.00001$

End If

Select Case FormulaChoice

Case 1 'Holladay I

RIndexCorn $=4 / 3$

R_th $=0.25$

Const Pc2 $=0.05$

$\mathrm{ALo}=. \mathrm{AxLu}+\mathrm{R}$ th $-\mathrm{Pc} 2$

$\mathrm{ELPo}=$ HolladayI(ELPpsnd, .AxLu, .AvKk)

MsgBox ELPo

Case 2 'HofferQ

RIndexCorn $=1.336$

$\mathrm{ALo}=. \mathrm{AxLu}$

$\mathrm{ELPo}=$ HofferQ(ELPpsnd, .AxLu, .AvKk) 


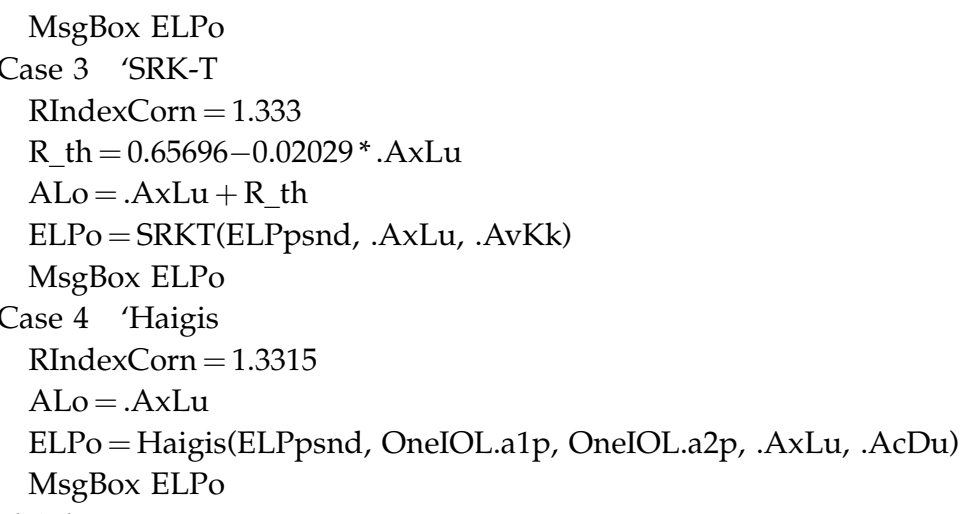

End Select

$\mathrm{Ko}=. \mathrm{AvKk}^{*}((\mathrm{RIndexCorn}-1) /(1.3375-1)) \quad$ 'converts to optical average $\mathrm{K}$

$X=1336 /((1000 /((1000 / . \operatorname{Ref})-$. Vrtx $))+$ Ko $)$

IOLtargetPower $=$ GivePower $($ ELPo, ALo, X)

IOLtargetPower $=$ AdjustTargetIOL(IOLtargetPower)

For Counter $=0$ To 12 'changing element of Array for each offset

Offset $=3-(0.5 *$ Counter $)$

PowerArray (Counter, 0) $=$ IOLtargetPower + Offset 'fill 1st column with IOL powers

PowerArray $($ Counter, 1) $=$ GiveSEq((IOLtargetPower + Offset), ALo, Ko, ELPo, .Vrtx $)$

Next Counter

End With

End Sub

Public Function GivePower(ByVal ELP As Double, ByVal AL As Double, ByVal Xconst As Double) As Double

If $(\mathrm{ELP}=0)$ Then

GivePower $=0$

Else

GivePower $=1336 *((1 /(\mathrm{AL}-\mathrm{ELP}))-(1 /($ Xconst $-\mathrm{ELP})))$

End If

End Function

Public Function HolladayI(ByVal ELPconst As Double, ByVal ALu As Double, ByVal Kk As Double) As Double Dim $r$ As Double, rag As Double, AG As Double, ACD As Double, SF As Double

$\mathrm{r}=1000 *(1.3375-1) / \mathrm{Kk}$

If $\mathrm{r}<7$ Then

rag $=7$

Else

$\mathrm{rag}=\mathrm{r}$

End If

$\mathrm{AG}=12.5^{*}(\mathrm{ALu} / 23.45)$

If $\mathrm{AG}>13.5$ Then

$\mathrm{AG}=13.5$

End If

$\mathrm{ACD}=0.56+\operatorname{rag}-\operatorname{Sqr}\left(\left(\operatorname{rag}^{\wedge} 2\right)-\left(\mathrm{AG}^{\wedge} 2 / 4\right)\right)$

$\mathrm{SF}=\mathrm{ELPtoSF}(\mathrm{ELP}$ const $)$

HolladayI $=\mathrm{ACD}+\mathrm{SF}$

End Function

Public Function HofferQ(ByVal ELPconst As Double, ByVal ALu As Double, ByVal Kk As Double) As Double Dim M As Double, G As Double, ACD As Double, K As Double

$\mathrm{ACD}=$ AtoACD(ELPtoA(ELPconst)

If $\mathrm{ALu}>31$ Then

$\mathrm{ALu}=31$

End If

If ALu < 18.5 Then 


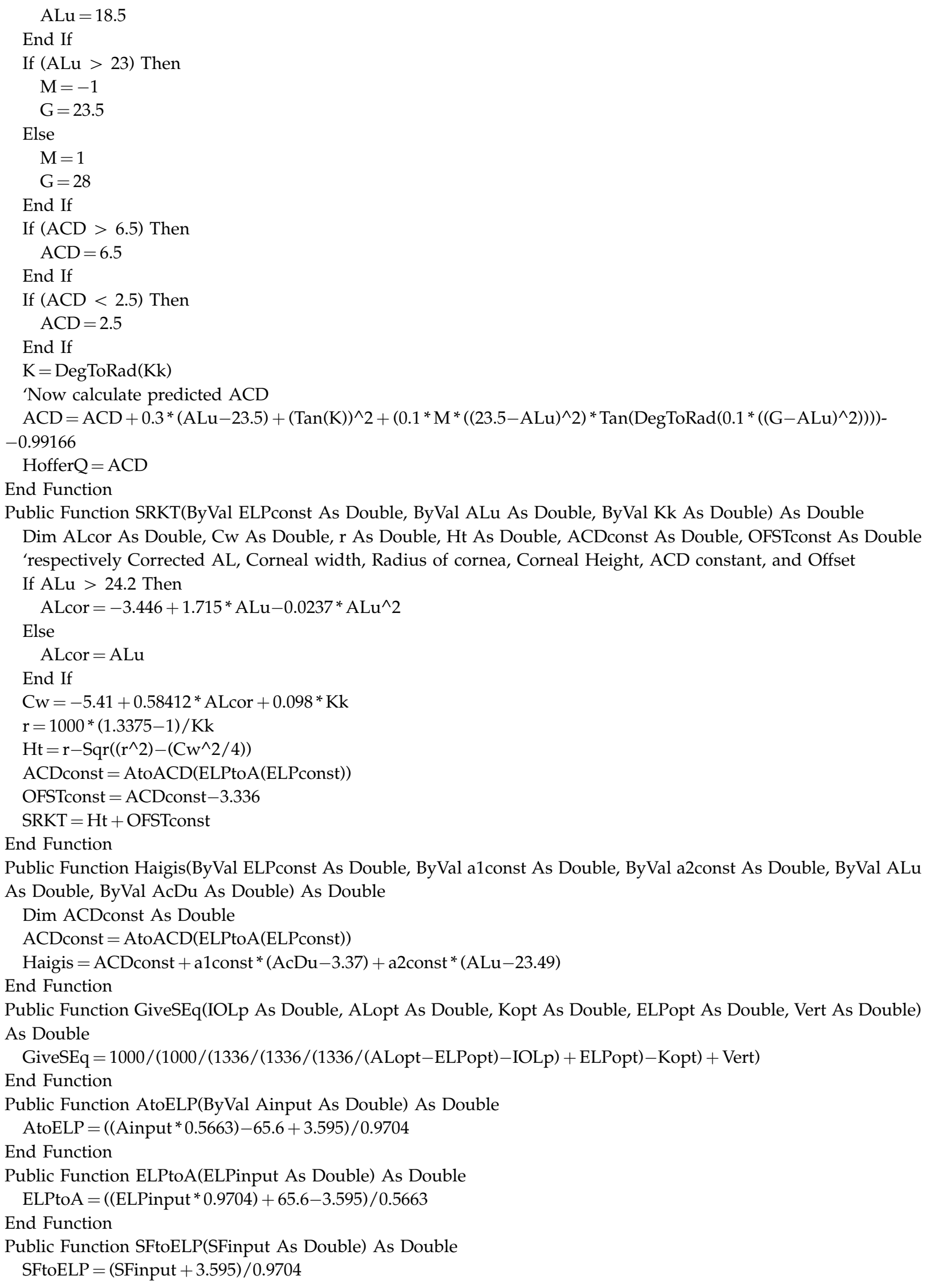


End Function

Public Function ELPtoSF(ELPinput As Double) As Double

$\mathrm{ELPtoSF}=\left(\right.$ ELPinput $\left.{ }^{*} 0.9704\right)-3.595$

End Function

Public Function AtoACD(Ainput As Double) As Double AtoACD $=($ Ainput $* 0.62467)-68.747$

End Function

Public Function ACDtoA(ACDinput As Double) As Double $\mathrm{ACD}$ toA $=(\mathrm{ACDinput}+68.747) / 0.62467$

End Function

Public Function DegToRad(ByVal Deg As Double) As Double Const PI $=3.14159265358979$

DegToRad $=$ Deg * $(P I / 180)$

End Function

Public Function AdjustTargetIOL(TargetIOL As Double) As Double

Dim Addfactor As Double

Const STEP $=0.25$

Dim IntPart As Integer

IntPart $=$ Int (TargetIOL)

Select Case (TargetIOL-IntPart)

Case Is $<=$ STEP

Addfactor $=0$

Case Is $<=$ STEP $^{*} 3$

Addfactor $=\mathrm{STEP}^{*} 2$

Case Else

Addfactor $=1$

End Select

AdjustTargetIOL $=$ IntPart + Addfactor

End Function 